\title{
On the establishment of the Takagi Lectures
}

\section{Toshiyuki Kobayashi}

Published online: 28 March 2007

(C) The Mathematical Society of Japan and Springer 2007

\begin{abstract}
The Takagi Lectures are the first series of lectures in mathematics to be crowned with a Japanese mathematician's name. The author provides some historical background of the Takagi Lectures.
\end{abstract}

Keywords and phrases: Takagi Lectures, Japanese Journal of Mathematics, Teiji Takagi

Mathematics Subject Classification (2000): 01A27, 01A61

In this issue of the Japanese Journal of Mathematics (JJM) there are three papers [1-3] that are the outcome of the 1st Takagi Lectures by the distinguished speakers S. Bloch, P.-L. Lions, and S. Smale. The paper [5] of C. Voisin, another Takagi Lecturer, is planned to be published in the next issue. The Takagi Lectures are the first series of lectures in mathematics to be crowned with a Japanese mathematician's name, and here I would like to provide some historical background.

In 2004, the Mathematical Society of Japan (MSJ) was faced with a crisis involving the Japanese Journal of Mathematics (JJM 2nd series), when the publisher, Kinokuniya, declared their intention to stop publishing the journal. Moreover, the expanded committee for the former JJM, consisting of representatives of the fourteen supporting journals, ended with an expression of pessimism about the future of JJM. At the end of 2004, I was in a meeting led by Professor Yasuo Morita (the former president of MSJ) about the future of JJM, partly because I was the editor in chief of another official journal of MSJ and was also a member of the board of trustees of MSJ at that time. In the face of the majority opinion of that meeting - that there was no way to continue publishing the journal any longer - I insisted that we should respect the fact

T. KOBAYASHI

Research Institute for Mathematical Sciences, Kyoto University Kyoto 606-8502, Japan (e-mail: toshi@kurims.kyoto-u.ac.jp) 
that JJM had the longest history among Japanese mathematics journals that have been published continuously in European languages (in the case of JJM, since 1924). We finally decided that we should continue the name of JJM but with new aims and a new scope. Thus, we decided to relaunch JJM in its 3rd series to publish research survey articles of the highest quality, which would be upheld by the peer-review process. I was then assigned to develop the 3 rd series. It was a daunting task for me to relaunch the journal within one year.

In the process of creating a plan for the future of JJM, I saw that we needed to have a driving force in order to continue publishing research surveys of the highest quality. For this purpose, I believed that it would be ideal to hold distinguished lectures periodically. The idea was that the audience would listen with interest and attention to the research surveys presented at the lectures, and, afterward, reading the resulting papers, they would recall the lectures vividly. This proposal was supported strongly at a meeting of the board of trustees of MSJ, and the suggestion was made to name this periodic event the Takagi Lectures in honor of Professor Teiji Takagi (1875-1960), considered as the founder of the Japanese School of Modern Mathematics (see [4]). In March 2006, with approval having been given by Professor Takagi's family, the MSJ General Assembly finalized the establishment of the Takagi Lectures. Incidentally, the assembly was held at the annual conference of the MSJ, where the first issue of the 3rd series of the Japanese Journal of Mathematics had just been released.

Subsequently, the editors of the JJM were assigned to become the organizing committee for the Takagi Lectures, and fortunately we were able to announce proudly the distinguished program shown on p.148. In November 2006, the 1st Takagi Lectures were held in a beautiful autumn setting in Kyoto, and they were very successful. Furthermore, the MSJ decided to use the surplus from the International Congress of Mathematicians, which was held in Kyoto in 1990, to support the funding of the Takagi Lectures.

I would like to take this opportunity to thank all those who have supported our endeavors. I hope that the Takagi Lectures will gain the respect of a worldwide audience and will continue to promote future progress in mathematics, in cooperation with the editors and contributors of the Japanese Journal of Mathematics.

\section{References}

[1] S. Bloch, Motives associated to graphs. Japan. J. Math., 2 (2007), 165-196.

[2] F. Cucker and S. Smale, On the mathematics of emergence. Japan. J. Math., 2 (2007), $197-$ 227.

[3] J.-M. Lasry and P.-L. Lions, Mean field games. Japan. J. Math., 2 (2007), 229-260.

[4] K. Miyake, Teiji Takagi, Founder of the Japanese School of Modern Mathematics. Japan. J. Math., 2 (2007), 151-164.

[5] C. Voisin, Some aspects of the Hodge conjecture, submitted to Japan. J. Math. 


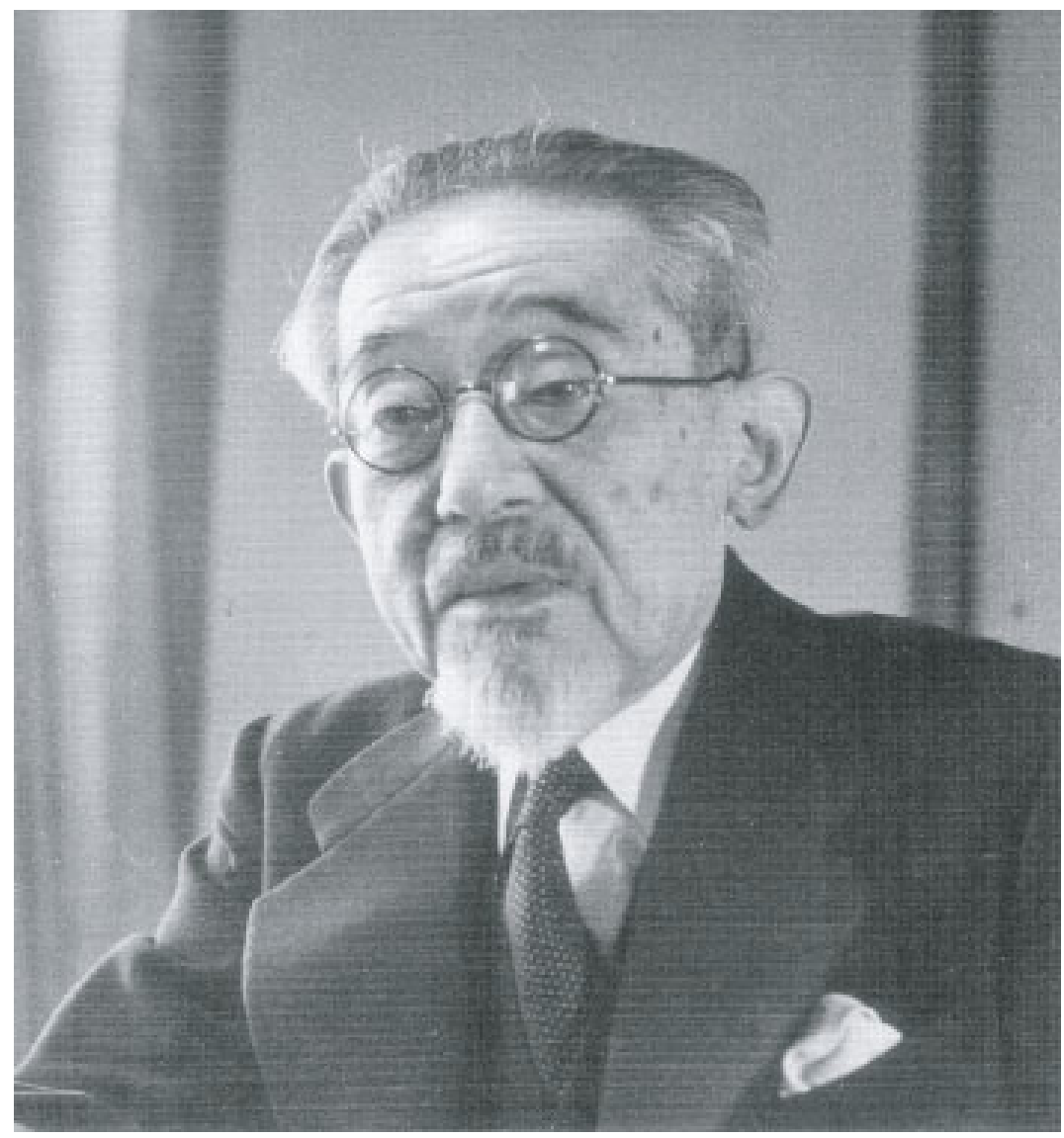

Teiji Takagi (1875-1960)

\section{Biography of Teiji Takagi*}

\begin{tabular}{|c|c|}
\hline 1875.4.21 & Born in Gifu Prefecture, Japan \\
\hline 1894 & Entered the Department of Mathematics, Imperial University of Tokyo \\
\hline 1897 & Entered the Graduate School of the same university \\
\hline 1898-1901 & Studied in Berlin and Göttingen \\
\hline 1903 & $\begin{array}{l}\text { Received the degree of Doctor of Science from the Imperial University } \\
\text { of Tokyo }\end{array}$ \\
\hline 1904 & Appointed Professor at the Imperial University of Tokyo \\
\hline 1920 & Published his main paper on the class field theory \\
\hline 1925 & Elected Member of the Imperial Academy of Japan \\
\hline 1936 & Served on the 1st Fields Medal Committee \\
\hline 1938 & Published the book A Course on Analysis (in Japanese) \\
\hline 1940 & Received Culture Medal \\
\hline 1960.2.28 & Died at the Hospital of Tokyo University \\
\hline
\end{tabular}

\footnotetext{
* extracted from "Chronological synopsis of the life of Teiji Takagi" by S. Iyanaga, In: Teiji Takagi Collected Papers, Second Enlarged Edition, Springer-Verlag Tokyo, 1990.
} 


\section{Program of the first Takagi Lectures}

Lecture Hall (Room No. 420),

Research Institute for Mathematical Sciences,

Kyoto University, Kyoto, Japan

\section{November 25, 2006}

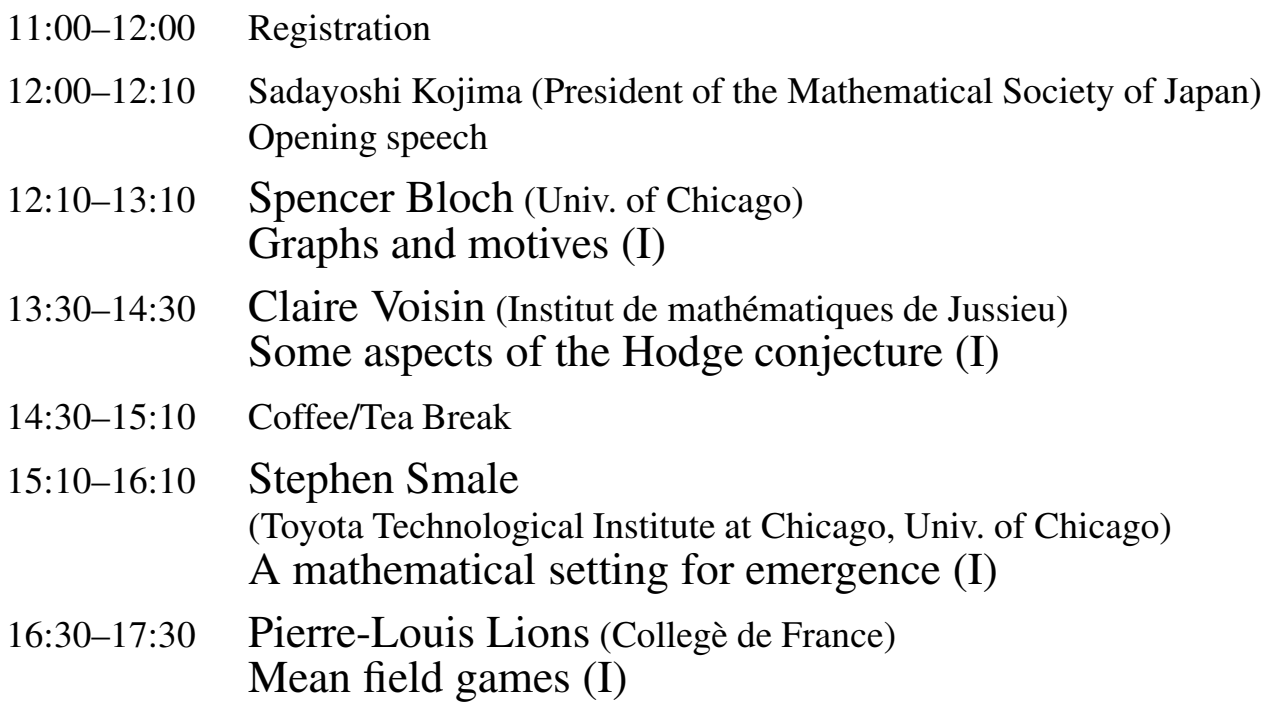

November 26, 2006

09:30-10:30 Spencer Bloch (Univ. of Chicago)

Graphs and motives (II)

10:50-11:50 Claire Voisin (Institut de mathématiques de Jussieu)

Some aspects of the Hodge conjecture (II)

11:50-13:30 Lunch Break

13:30-14:30 Stephen Smale

(Toyota Technological Institute at Chicago, Univ. of Chicago)

A mathematical setting for emergence (II)

14:50-15:50 Pierre-Louis Lions (Collegè de France)

Mean field games (II)

16:00-17:00 Workshop closure with drinks

\section{Organizing Committee}

Y. Kawahigashi

T. Kobayashi

H. Nakajima

K. Ono

T. Saito 